\title{
A COUNTEREXAMPLE TO A VITALI TYPE THEOREM WITH RESPECT TO HAUSDORFF CONTENT
}

\author{
MARK MELNIKOV AND JOAN OROBITG
}

(Communicated by Andrew M. Bruckner)

\begin{abstract}
Mateu and Orobitg proved (in Lipschitz approximation by harmonic functions and some applications to spectral synthesis, Indiana Univ. Math. J. 39 (1990)) that given $\lambda>1$ and $d-1<\alpha \leq d$ there exist constants $C$ and $N$ (depending on $\lambda$ and $\alpha$ ) with the following property:

For any compact set $K$ in $\mathbb{R}^{d}$ one can find a (finite) family of balls $\left\{B\left(x_{i}, r_{i}\right)\right\}$ such that (i) $K \subset \cup B\left(x_{i}, r_{i}\right)$, (ii) $\sum r_{i}^{\alpha} \leq C M^{\alpha}(K), M^{\alpha}$ denoting the $\alpha$-dimensional Hausdorff content, and (iii) the dilated balls $\left\{B\left(x_{i}, \lambda r_{i}\right)\right\}$ are an almost disjoint family with constant $N$.

In this paper we prove that such a result is false for $\alpha \leq d-1$.
\end{abstract}

\section{Statement of the Result}

Given a subset $E$ of $\mathbb{R}^{d}$ one defines the $\alpha$-dimensional Hausdorff content of $E$ by

$$
M^{\alpha}(E)=\inf \sum_{j} l\left(Q_{j}\right)^{\alpha},
$$

where the infimum is taken over all countable coverings of $E$ by cubes $Q_{j}$ with sides of length $l\left(Q_{j}\right)$ and parallel to the coordinate axes.

In [MO] the $\operatorname{Lip}(s)$ approximation problem by harmonic functions, $0<s<$ 1 , was solved using the following [MO, Lemma 2.3].

Covering lemma. Given $\lambda>1$ and $d-1<\alpha \leq d$ there exist constants $C$ and $N$ (depending on $\lambda$ and $\alpha$ ) such that for any compact set $K$ in $\mathbb{R}^{d}$ one can find a finite family of cubes $\left\{Q_{i}\right\}$ satisfying.

(i) $K \subset \cup Q_{i}$,

(ii) $\sum_{i} l\left(Q_{i}\right)^{\alpha} \leq C M^{\alpha}(K)$,

(iii) $\left\{\lambda Q_{i}\right\}$ is an almost disjoint family with constant $N$.

Remark. If $M^{\alpha}(K)=0$ then (ii) should be replaced by $\sum_{j} l\left(Q_{i}\right)^{\alpha} \leq \varepsilon$, where $\varepsilon>0$ has been fixed in advance.

It is said that a family $\left\{E_{j}\right\}$ of subsets of $\mathbb{R}^{d}$ is almost disjoint with constant $N$ whenever each $x \in \mathbb{R}^{d}$ belongs to at most $N$ sets $E_{j}$. If $Q$ is a cube then $\lambda Q$ denotes the cube of the same center and side length $\lambda l(Q)$.

Received by the editors July 31, 1991 and, in revised form, November 4, 1991.

1991 Mathematics Subject Classification. Primary 28A12, 28 A75.

The second author was supported in part by the grant PB89-0311, DGICYT. 
In this paper we prove that the above result is false for $0<\alpha \leq d-1$. More precisely

Theorem. Given constants $\lambda>1, C>1,0<\alpha \leq d-1$, and a positive integer $N$ there exists a compact set $K=K(\lambda, C, \alpha, N)$ with $M^{\alpha}(K)>0$ such that no family of cubes $\left\{Q_{i}\right\}$ can be found satisfying simultaneously:

(i) $K \subset \cup Q_{i}$,

(ii) $\sum_{i} l\left(Q_{i}\right)^{\alpha} \leq C M^{\alpha}(K)$,

(iii) $\left\{\lambda Q_{i}\right\}$ is an almost disjoint family with constant $N$.

This proposition answers in the negative a question raised in [MV] in connection with BMO approximation by harmonic functions in the plane. A positive answer to the case $\alpha=d-1$ would have given a suitable extension of their results to higher dimensions. Thus, new ideas seem to be required to obtain BMO approximation results by harmonic functions in $\mathbb{R}^{d}, d \geq 3$.

In order to free our exposition of notational complications we will only present the proof of the theorem for $d=2$ and $\alpha=1$. The general case follows from the same basic ideas with simple technical modifications.

Before coming to the proof of the theorem, some reductions are in order. First let us check that it is enough to prove the theorem only for a fixed $\lambda>1$. Assume that one has a compact set $K$ and a family of squares $\left\{Q_{i}\right\}$ satisfying (i), (ii), and (iii) for some $\lambda_{1}>1, C>1$, and $N$. Let $\lambda_{2}>\lambda_{1}$ and let $s$ be the integer part of $1+\left(\lambda_{2}-1\right) /\left(\lambda_{1}-1\right)$. We split each square $Q_{i}$ into $s^{2}$ squares of side length $s^{-1} l\left(Q_{i}\right), Q_{i}=\bigcup_{j} P_{i}^{j}$. Then the family $\left\{P_{i}^{j}\right\}$ satisfies (i), (ii), and (iii) with $\left\{Q_{i}\right\}$ replaced by $\left\{P_{i}^{j}\right\}$ and $\lambda_{1}, C$, and $N$ replaced by $\lambda_{2}, s C$, and $s^{2} N$, respectively. So, one can assume $\lambda=3$.

Likewise, it will be more convenient to work with a special type of squares instead of all squares with sides parallel to the coordinate axes. We will consider tryadic squares. A tryadic square has a representation $\left[j 3^{n},(j+1) 3^{n}\right] \times$ $\left[k 3^{n},(k+1) 3^{n}\right]$, where $j, n$, and $k$ are integers. We remark that if $Q$ and $P$ are tryadic squares then either they have disjoint interiors or one contains the other. This fact will be used without further comment in our discussion.

The tryadic 1-dimensional Hausdorff content of a set $E$ of $\mathbb{R}^{2}$ is defined by

$$
M_{t}^{1}(E)=\inf \sum_{j} l\left(Q_{j}\right)
$$

where now the infimum is taken over all countable coverings of $E$ by tryadic squares $Q_{j}$. Obviously, $M^{1}(E) \leq M_{t}^{1}(E)$ for all sets $E$. Given a square $Q$, let $Q_{i}$ be the tryadic squares that intersect $Q$ with side length $3^{j}, 3^{j} \leq l(Q) / 2<$ $3^{j+1}$, then

(a) $Q \subset \cup Q_{i}$

(b) $\bigcup 3 Q_{i} \subset 3 Q$.

Condition (a) gives $M_{t}^{1}(E) \leq 9 M^{1}(E)$. From condition (b) it follows that if we have a family of squares covering a set $E$ such that their triples are almost disjoint with constant $N$, then we can find a family of tryadic squares (of comparable size) covering the same set such that their triples are almost disjoint with constant $9 N$. 
By the above remarks the only task left is to prove the next

Proposition. Given a constant $C>1$ and a positive integer $N$, there exists a compact set $K=K(C, N)$ with $M_{t}^{1}(K)>0$ such that no family of tryadic squares $\left\{Q_{i}\right\}$ can be found satisfying simultaneously:

(i) $K \subset \cup Q_{i}$,

(ii) $\sum_{i} l\left(Q_{i}\right) \leq C M_{t}^{1}(K)$,

(iii) $\left\{3 Q_{i}\right\}$ is an almost disjoint family with constant $N$.

\section{PROOF OF THE PROPOSITION}

Having fixed $C>1$ and a positive integer $N$, we will construct a compact subset $K$ of the unit square $I=[0,1] \times[0,1]$ such that it will be impossible to find a family of tryadic squares satisfying (i), (ii), and (iii) simultaneously. Our procedure is based on the next lemma.

Main lemma. Given a tryadic square $Q$ and a constant $C>1$, let $j=$ $[(C-1) / 2]+1$ and let $m$ be the first integer such that $3^{m} \geq 3 C$. We put $\alpha=\alpha(C, l(Q))=l(Q) 3^{-j-m}$. Then there exists a positive integer $N(\alpha) \geq$ $3 \mathrm{Cl}(Q) / \alpha$ such that for each family of tryadic squares $\left\{Q_{i}\right\}$ satisfying

(1) $Q \subset \cup Q_{i}$,

(2) $\sum_{i} l\left(Q_{i}\right) \leq C l(Q)$,

(3) $l\left(Q_{i}\right) \geq 3 \alpha$ if $Q_{i} \cap Q^{c} \neq \varnothing$,

one can find $N(\alpha)$ tryadic squares of side length $\alpha$ contained in $\left(\cup 3 Q_{i}\right) \backslash\left(\cup Q_{i}\right)$.

Assume that the main lemma has been proved and let us proceed to the proof of the proposition. Without loss of generality we can suppose the constant $C$ is integer and multiple of 3.

Set $C_{1}=3 C, \delta_{1}=\left(3 C_{1}\right)^{-1}$, and for $i=1, \ldots, N$ write

$$
\alpha_{i}=\alpha\left(C_{i}, \delta_{i}\right)=\delta_{i} 3^{-j_{i}-m_{i}}, \quad \delta_{i+1}=\frac{\delta_{i}}{3} \alpha_{i}^{2}, \quad C_{i+1}=\frac{5}{4} \frac{1}{\delta_{i} \alpha_{i}} .
$$

Our compact set $K$ will be union of compact sets $K_{1}, K_{2}, \ldots, K_{N+1}$.

$K_{1}$ is the tryadic square in the center of the unit square $I$ with side length $\delta_{1}$ (see Figure 1).

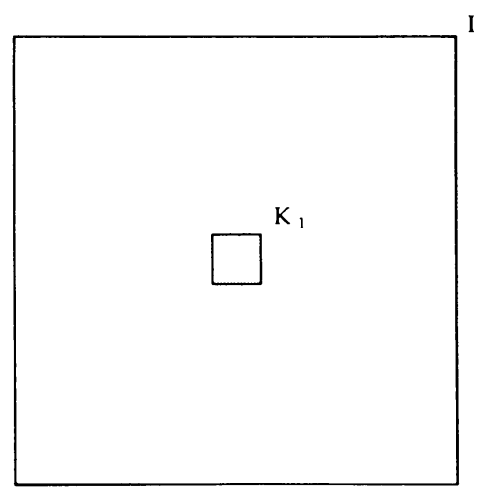

FIGURE 1 
Inside of each tryadic square of side length $\alpha_{1}$ in $I \backslash K_{1}$, consider the $n_{2}$ tryadic squares of side length $\delta_{2}$ with the same center and let $K_{2}$ be their union. Thus $M_{t}^{1}\left(K_{2}\right) \leq n_{2} \delta_{2}<\alpha_{1}^{-2} \delta_{2}=\delta_{1} / 3$.

If $i \in\{3, \ldots, N+1\}$ then $K_{i}$ is inductively defined as follows. $K_{i}$ is the union of all tryadic squares of side length $\delta_{i}$ concentric with the tryadic squares of length $\alpha_{i-1}$ in $I \backslash \bigcup_{j=1}^{i-1} K_{j}$. Let $n_{i}$ be the number of them. Thus, $M_{t}^{1}\left(K_{i}\right) \leq n_{i} \delta_{i}<\alpha_{i-1}^{-2} \delta_{i}=\delta_{i-1} / 3$.

Define

$$
K=K_{1} \cup K_{2} \cup \cdots \cup K_{N+1},
$$

so that $M_{t}^{1}(K) \leq \delta_{1}+\delta_{1} / 3+\cdots+\delta_{N} / 3<\frac{3}{2} \delta_{1}$.

We are now going to check that $K$ satisfies the desired properties.

Let $\mathscr{Q}=\left\{Q_{i}\right\}$ be some fixed family of tryadic squares such that

(i) $K \subset \bigcup Q_{i}$,

(ii) $\sum l\left(Q_{i}\right) \leq C M_{t}^{1}(K)<C_{1} \delta_{1} \leq 1 / 2$.

We will show that there exists a point that belongs at least to $N+1$ sets of the family $\left\{3 Q_{i}\right\}$. In other words, $\left\{3 Q_{i}\right\}$ is not an almost disjoint family with constant $N$.

Define $\mathscr{F}=\left\{Q_{i} \in \mathscr{Q}: Q_{i}\right.$ กint $K_{1} \neq \varnothing$ or $\left.l\left(Q_{i}\right) \geq 3 \alpha_{1}\right\}$. Applying the lemma, we get $N\left(\alpha_{1}\right)$ tryadic squares $P$ with $l(P)=\alpha_{1}$ contained in $\bigcup_{\mathscr{F}} 3 Q_{i} \backslash \bigcup_{\mathscr{F}} Q_{i}$. For each of those squares $P$, one has two possibilities:

(I) $\sum_{Q_{i} \subset P} l\left(Q_{i}\right) \leq C_{2} \delta_{2}<\alpha_{1} / 2$, or

(II) $\sum_{Q_{i} \subset P} l\left(Q_{i}\right)>C_{2} \delta_{2}$.

If, for all squares $P$, (II) holds, then

$$
\sum_{i} l\left(Q_{i}\right) \geq N\left(\alpha_{1}\right) C_{2} \delta_{2} \geq \frac{3 C_{1} \delta_{1}}{\alpha_{1}} C_{2} \delta_{2}>C_{1} \delta_{1},
$$

where the second inequality follows from the lemma and the last from the definition of $\delta_{2}$ and $C_{2}$. Clearly the above inequality contradicts property (ii) Q . Therefore, there exists a tryadic square $P_{1}$ with side length $\alpha_{1}$ contained in $\bigcup_{\mathscr{G}} 3 Q_{i} \backslash \bigcup_{\mathscr{G}} Q_{i}$ and satisfying (I). Every point of $P_{1} \cap K$ belongs at least to 2 squares in the family $\left\{3 Q_{i}\right\}$.

Let $\mathscr{Q}^{1}=\left\{Q_{i} \in \mathscr{Q}: Q_{i} \subset P_{1}\right\}$. Since

(i) $K \cap P_{1} \subset \bigcup_{\mathscr{Q}_{1}} Q_{i}$,

(ii) $\sum_{Q^{1}} l\left(Q_{i}\right) \leq C_{2} \delta_{2}<l\left(P_{1}\right) / 2$,

we can now repeat the argument with $\mathscr{Q}$ replaced by $\mathscr{Q}^{1}$ and $K$ replaced by $K \cap P_{1}$

Repeating the above process, it becomes clear that we can find a point that belongs at least to $N+1$ sets of family $\left\{3 Q_{i}\right\}$. This completes the proof of the proposition.

\section{Proof of the main lemma}

We start with two simple remarks.

Remark 1. If one has $Q=\bigcup_{i} Q_{i}$, where $Q$ and $Q_{i}$ are tryadic squares, and one wants to study the set $\cup 3 Q_{i} \backslash Q$, then one should only consider squares $Q_{i}$ that intersect the boundary $\partial Q$ of $Q$. In other words, if $Q_{i}$ does not touch $\partial Q$ then $3 Q_{i} \subset Q$. 
Remark 2. Keeping the notation in Remark 1, let $\mathscr{B}=\left\{Q_{i}: Q_{i} \cap \partial Q \neq \varnothing\right\}$. It is a simple calculation to check that if $\sum l\left(Q_{i}\right) \geq 1$, where the sum is taken over all squares $Q_{i} \in \mathscr{B}$ such that $l\left(Q_{i}\right) \leq l(Q) 3^{-k}$, then $\sum_{i} l\left(Q_{i}\right) \geq(2 k+1) l(Q)$.

Let $\mathscr{Q}=\left\{Q_{i}\right\}$ be a family of squares satisfying the conditions (1), (2), and (3) of the lemma. Without loss of generality we can assume that these squares have disjoint interiors. Suppose that there does not exist any $Q_{i} \in \mathscr{Q}$ that contains $Q$ and subdivide $\mathscr{Q}$ into two families $\mathscr{Q}^{1}=\left\{Q_{i} \in \mathscr{Q}: Q_{i} \subset Q\right\}$ and $\mathscr{Q}^{2}=\mathscr{Q} \backslash \mathscr{Q}^{1}$. We have

$$
\sum_{Q_{i} \in Q^{1}} l\left(Q_{i}\right)=A l(Q)
$$

where $A$ is a constant less than $C$. Consider $\mathscr{B}=\left\{Q_{i} \in \mathscr{Q}: Q_{i} \cap \partial Q \neq \varnothing\right\}$ and define $l=[(A-1) / 2]+1$. Then

$$
\sum_{Q_{i} \in \mathscr{B}, l\left(Q_{i}\right) \leq l(Q) 3^{-l}} l\left(Q_{i}\right)<l(Q),
$$

because otherwise from Remark 2 we would get

$$
\sum_{Q_{i} \in Q^{1}} l\left(Q_{i}\right) \geq(2 l+1) l(Q)>A l(Q),
$$

which contradicts (1).

We can conclude that the set (see Figure 2)

$$
\bigcup_{Q^{1}} 3 Q_{i} \backslash \bigcup_{Q^{1}} Q_{i}
$$

contains at least $2 \cdot 3^{l-1}$ squares $P$ with $l(P)=l(Q) 3^{1-l}$ (touching $\partial Q$ ) and

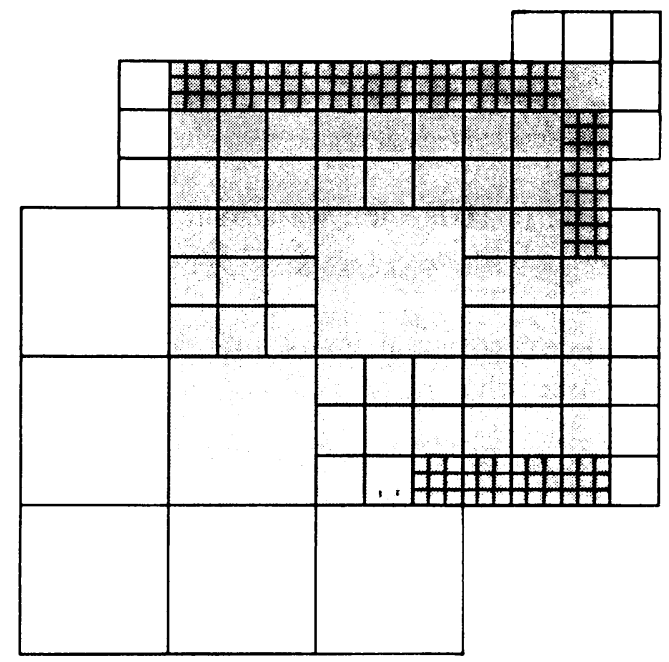

FIGURE 2 
Q

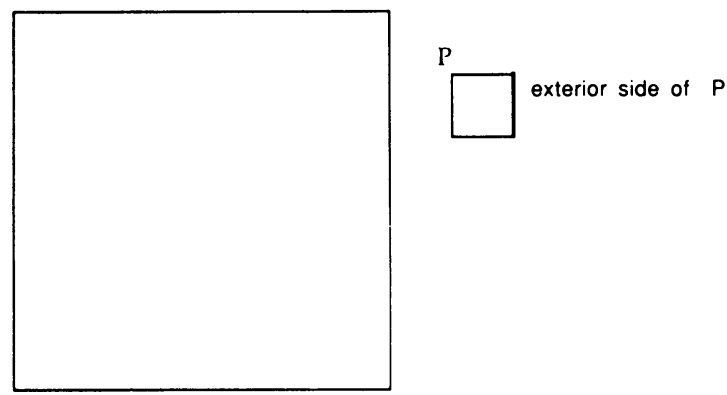

FIGURE 3

the union of them has tryadic 1-dimensional Hausdorff content greater than $2 l(Q)$, because from (2) it follows that

$$
\sum_{Q_{i} \in \mathscr{B}, l\left(Q_{i}\right) \geq l(Q) 3^{-l}} l\left(Q_{i}\right) \geq \frac{5}{3} l(Q) .
$$

Denote this family of squares by $\mathscr{P}$.

Note that if for some $Q^{*} \in \mathscr{Q}$ we had $Q \subset Q^{*}$, then setting $\mathscr{Q}^{1}=\left\{Q^{*}\right\}$ we would immediately reach the same conclusion.

We are interested in the set $\bigcup_{\mathscr{Q}} 3 Q_{i} \backslash \bigcup_{\mathscr{Q}} Q_{i}$, and so far we have only considered (3). We must now look at the squares from the family $\mathscr{Q}^{2}$. Given $Q_{i} \in \mathscr{Q}^{2}$ we have four cases:

(I) $Q_{i}$ and $P$ have disjoint interiors for all $P \in \mathscr{P}$.

(II) $l\left(Q_{i}\right) \geq l(Q) 3^{1-l}$.

(III) There exists some $P \in \mathscr{P}$ such that $Q_{i} \subset P$ and $\partial Q_{i}$ touches the exterior side of $P$, that is, the side of $P$ that lies further from $Q$ (see Figure 3).

(IV) There exists some $P \in \mathscr{P}$ such that $Q_{i} \subset P$ and $\partial Q_{i}$ does not touch the exterior side of $P$.

If $Q_{i}$ satisfies (I) or (II) or (III) then we add it to $\mathscr{Q}^{1}$ and still the property mentioned after $(3)$ holds, that is, $\bigcup_{\mathscr{Q}^{1}} 3 Q_{i} \backslash \bigcup_{\mathscr{Q}^{1}} Q_{i}$ contains a family $\mathscr{P}$ of squares $P$ with $l(P)=l(Q) 3^{1-l}$, the number of them is at least $2 \cdot 3^{l-1}$ and their union has tryadic 1-dimensional Hausdorff content bigger than $2 l(Q)$.

Therefore, we can assume that if $Q_{i} \in \mathscr{Q}^{2}$ then

(a) $Q_{i}$ is contained in some $P \in \mathscr{P}$ and $l\left(Q_{i}\right) \leq l(Q) 3^{-l}$;

(b) $Q_{i}$ does not touch the exterior side of $P$.

Moreover, recall that we have

(c) $l\left(Q_{i}\right) \geq 3 \alpha$;

(d) $\sum_{Q^{2}} l\left(Q_{i}\right) \leq(C-A) l(Q)$. 


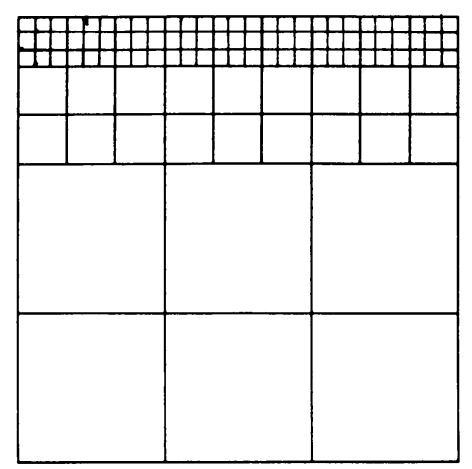

FIGURE 4

Now we are able to prove that the number $N(\alpha)$ of squares of side length $\alpha$ inside

$$
\left(\bigcup_{Q^{1}} 3 Q_{i}\right) \backslash\left(\bigcup_{Q^{1}} Q_{i}\right) \backslash\left(\bigcup_{Q^{2}} Q_{i}\right)
$$

is bigger than $3 \mathrm{Cl}(Q) \alpha^{-1}$. In fact, we are going to see that these squares are inside

$$
\bigcup_{\mathscr{D}} P \backslash \bigcup_{\mathscr{Q}^{2}} Q_{i}
$$

Owing to properties of family $\mathscr{P}$ and conditions (a), (b), and (c) of family $\mathscr{Q}^{2}$, it is sufficient (and obviously necessary) to prove that the area of (4) is not less than $2 l(Q)^{2} 3^{-j}$ (because then $N(\alpha)=\alpha^{-2}$ Area $=\alpha^{-1} 2 l(Q) 3^{m}>$ $\left.\alpha^{-1} 3 C l(Q)\right)$.

The area of the set (4) will be minimized when the squares $Q_{i}$ are as big as possible, and this will happen whenever in each square $P$ we take 6 squares of side length $l(Q) 3^{-l}, 6$ squares of side length $l(Q) 3^{-1-l}$, and so on until the squares of size $l(Q) 3^{1-s-l}$ (see Figure 4).

Consequently, $\sum l\left(Q_{i}\right) \geq 4 s l(Q)$ and by condition (d) we have $s \leq$ $(C-A) / 4$. Therefore

$$
\text { Area } \geq 2 \cdot 3^{l-1} l(Q) 3^{1-l} l(Q) 3^{1-s-l} \geq 2 l(Q)^{2} 3^{1-l-s} \geq 2 l(Q)^{2} 3^{-j},
$$

where the last inequality follows from

$$
s+l-1 \leq \frac{C-A}{4}+\frac{A-1}{2}=\frac{C+A-2}{4} \leq \frac{C-1}{2} \leq j .
$$

\section{ACKNOWLEDGMENT}

Thanks are due to J. Mateu and J. Verdera for useful conversations on the subject of this paper. The first author wishes to express his gratitude to the Centre de Recerca Matemàtica and to the Department of Mathematics of Universitat Autònoma de Barcelona for its warm hospitality. 


\section{REFERENCES}

[MO] J. Mateu and J. Orobitg, Lipschitz approximation by harmonic functions and some applications to spectral synthesis, Indiana Univ. Math. J. 39 (1990), 703-736.

[MV] J. Mateu and J. Verdera, BMO harmonic approximation in the plane and spectral synthesis for Hardy Sobolev spaces, Rev. Mat. Iberoamericana 4 (1988), 291-318.

Mechanic Mathematical Faculty, TfFa, Moscow State University, Moscow 117234 , RUSSIA

Current address: Departament de Matemátiques, Universitat Autònoma de Barcelona, 08193 Bellaterra, Spain

Departament de Matemàtica Aplicada II, Universitat Politècnica de Catalunya, Pau Gargallo 5, 08028 Barcelona, Spain

E-mail address: orobitg@ma2.upc.es 